\title{
Spasticity in Patients with Severe Acquired Brain Injury: An Inpairment That Is Poorly Understood and Poorly Measured
}

\author{
Maurizio Falso* \\ From the Section of Neurological Rehabilitation, Clinical Institute Città di Brescia, Brescia, Italy
}

*Corresponding author: Maurizio Falso, From the Section of Neurological Rehabilitation, Clinical Institute Città di Brescia, Brescia, Italy, E-mail: falsomaurizio@libero.it

Received Date: September 28, 2015

Accepted Date: January 13, 2016

Published Date: January 19, 2016

\begin{abstract}
Objective: to investigate any parametric and non-parametric correlations that might influence the development of spasticity in 27 patients affected by SBI in order to optimize their clinical and rehabilitative approach.

Design: an observational and investigational study.

Patients: 27 patients (14 males, 13 females; mean age $65 \pm 15 y$ ), affected by severe acquired brain injury and recovered in our Neurological Rehabilitation Section from February to October 2009.

Methods: in accordance with our clinical study inclusion criteria (GCS $<$ or $=8, \mathrm{LCF}<$ or $=3$, DRS $<$ or $=22)$, patients were divided in 4 observational groups (post-stroke group, post-haemorrhagic group, post-anoxic group, post-traumatic group) and evaluated under an epidemiological, clinical and functional point of view. All patients had been undergoing same physiotherapy during the recovery and observational period, 6 days a week, in 2 hour session, consisting in passive limb kinesis, neurodynamic limb exercises and postural control exercises.

Results: $85 \%$ of patients recruited developed an upper and/or lower limb spasticity, never demonstrated in other clinical evidences. We observed that a) post-haemorrhagic group showed a higher incidence of limb spasticity b) spasticity will develop earlier in poststroke and traumatic patients c) spasticity severity is stronger in post-anoxic and traumatic brain injury. These parametric data were not statistically significant $(\mathrm{p}>0.05)$. All patients received drug and physiotherapic treatment but only $30 \%$ of them will not develop limb spasticity. In line with the non- parametric statistical analysis, no correlation between the development of limb spasticity and nutritional and co-morbidity patients status was observed.

Conclusions: the main finding of our study is that post-anoxic and TBI lesions have severe clinical picture with more bilateral and severe limb spasticity. We also observed that post-haemorrhagic patients develop rapidly spasticity mainly in the limbs and mainly if the lesion involves the subcortical nuclei, but these findings are not in agreement with the recent literature. Treating limb spasticity we noted that rehabilitative approach was more successful in patients with haemorrhagic lesion than in patients affected by post-anoxic state or TBI. In spite of the described conclusions, the definition of spasticity is not well known and investigated and represents an important step in order to optimize the clinical and rehabilitative approach of patients with SBI and affected by spasticity and pain related syndrome.
\end{abstract}

Keywords: Spasticity; Severe brain injury; Outcome assessment

Citation: Falso, M. Spasticity in Patients with Severe Acquired Brain Injury: An Inpairment That Is Poorly Understood and Poorly Measured. (2016) Int J Neurol Brain Disord 3(1): 6- 10. 


\section{Introduction}

Spasticity is defined as a "motor disorder characterized by a velocity dependent increase in tonic stretch reflexes (muscle tone) and increased tendon jerks resulting from disinhibition of stretch reflex"[1,2]. To date, there is no clinical advanced evidence about the pathophysiological mechanisms underlying the development of spasticity and the complex clinical and rehabilitative management of patients affected by vegetative state secondary to severe acquired brain injury ${ }^{[3,4]}$. In the last years, different interpretations of this positive symptoms of the UMNS were given. The North American Task Force for Childhood Motor Disorders defined spasticity as 'a velocity dependent increase in hypertonia with a catch when a threshold is exceeded ${ }^{[5]}$. Moreover, the members of the SPASM consortium suggested that the definition spasticity can be interpreted as "a disordered sensory-motor control resulting from an upper motor neuron lesion, presenting as intermittent or sustained involuntary activation of muscles"[6]. Each research into spasticity need to establish valid and reliable outcome measures. In the clinical practice, measurement and treatment approach is vast and all measurements developed for the lower limb have also been adapted for treating the upper $\operatorname{limb} b^{[3]}$.

The aim of this interventional evaluation study was to explore the meaning of spasticity secondary to severe brain injury by investigating any parametric and non-parametric correlations that might influence the development of this positive symptom in SBI patients, in order to optimize their clinical and rehabilitative approach.

\section{Methods}

\section{Participants}

We recruited 27 patients (14 males, 13 females; mean age $65 \pm 15 y$ ), affected by severe acquired brain injury and recovered in our Neurological Rehabilitation Section from February to October 2012. In accordance with our "study inclusion criteria" (GCS $<$ or $=8, \mathrm{LCF}<$ or $=3$, DRS $<$ or $=22$ ), we recruited only patients affected by a "vegetative state". Patients were divided in 4 observational groups: a post-stroke group, a post-haemorrhagic group, a post-anoxic group and a post-traumatic group. Informed consent was obtained by patients' parents before beginning this observational clinical study.

\section{Observational clinical design}

Patients underwent to a standardized evaluation protocol at the beginning of the hospitalization and regularly each month after the last evaluation performed.

The protocol consists in two evaluation phases: the first, defined "beginning statement", which was compiled at the first patient's evaluation only; the second one was defined "actual statement" which was compiled at the beginning and at each outcome evaluation of the rehabilitative treatment course. Patients' neurological state was evaluated using the $\mathrm{GCS}^{[7]}$, an evaluation scale with a good interindividual variability and predictive reliability ${ }^{[8-9]}$. Functional outcome and patients' disability, as well as the evaluation of their persistent vegetative state, were performed using the GOS, LCF and DRS scales in line with the guidelines defined by the Consenus National Confer- ence $^{[10,11]}$.

In this observational clinical design, we preferred the GOS scale because universally adopted for the wellknown reliability and the simple use ${ }^{[10]}$; DRS demonstrated a good interexamination agreement ${ }^{[11]}$ and an higher sensibility than GOS as an outcome measure ${ }^{[12]}$; LCF is a large used scale in monitoring the responsively of patients evaluated. Evaluation of persistent vegetative state was performed using GOS, LCF and DRS in line with the guidelines defined by the Consensus National Conference $^{[11]}$. Spasticity was quantified using the Modified Ashworth Scale (MAS), which represent the gold standard in measuring the muscle tone in patients with $\mathrm{UMS}^{[13,14]}$, in spite of its limits in term of validity and reliability ${ }^{[15,16]}$. Patients' nutritional state was evaluated quantifying serum albumin level and $n^{\circ}$ of lymphocytes per $\mathrm{mm}^{3}$ of blood; we considered a malnutrition state if the $\mathrm{n}^{\circ}$ of lymphocytes $<1800 \mathrm{~mm}^{3}$ and serum albumin $<3.5 \mathrm{mg} /$ dl ${ }^{[17]}$. We also evaluated the eventual daily alcohol use during the anamnestic acquisition: in this study a daily use of alcohol $>2$ Units (20g of alcohol) was considered significant. The use of some kind of drug classes, underlying the time-course use or interruption of the pharmacological therapy adopted, was analysed.

\section{Rehabilitative treatment protocol}

All patients had been undergoing physiotherapy during the recovery and observational period, 6 days a week, in 2 hour session, in line with the GCS National Consensus Conference about the treatment protocol of patients affected by severe brain injury.

Our daily rehabilitation protocol consisted in:

- passive multijoint limb kinesis, 30min per day

- dailyneurodynamic limb exercises of the upper and lower limb

- postural control exercises

- side to side positioning, starting from the supine position, every 2 hours

- progressive vertical positioning using static bed and with a continuous monitoring of cardiovascular parameters (to do during the first two weeks of hospitalization)

- progressive conditioning to sitting position using disability-assistive chairs

- neurosensorial stimulations to optimize and recover patients' environment contact, relation faculties and to realize the optimal "milieu" for the communication recovery

- therapeutic exercise: stretching and rehabilitative techniques to reduce spasticity in line with the Bobath concept (to apply in spastic patients only).

Rehabilitation protocol also included the useof bed sore aids, positioning othersand breathing exercises for bronchial clearance and the conditioning to a progressive interruption of mechanical ventilation and recovery of spontaneous breath.

\section{Analysis of results}

All statistic data were performed using SPSS. A descriptive statistical analysis of the quantitative and qualitative parameters of mean (M) and SD was performed. We used chisquare to evaluate the correlation between the mentioned variables and spasticity. A p $<0.05$ was considered statistically significant. 


\section{Results}

\section{"Beginning statement" epidepiomological and behavioural evaluation}

The most representative class of age of patients recruited was between 66 and 75 years. 37\% of our patients had a primary school education, $33 \%$ a junior high school education, $18.5 \%$ a secondary school education and $11 \%$ a degree. $7.4 \%$ of patients used illegal drugs, $22 \%$ had an excessive use of alcohol and 15\% of our patients showed alcohol related problems. Time-course passed between brain damage event and beginning of rehabilitative treatment varied between 8 and 290 days (mean value $40 \pm 56$ days). $60 \%$ of patients were recovered between 10 and 30 days after brain damage event, $11 \%$ within 10 days after, $14 \%$ between 30 and 60 days after and 15\% after 2 months. Mean time recovery of patients recruited was about $164 \pm 57$ days Table 1.

Table 1: resume of our evaluation results.

\begin{tabular}{|c|c|c|c|c|c|c|c|}
\hline $\begin{array}{l}\text { Representative class of age of patients } \\
\text { recruited expressed in percentage (\%) }\end{array}$ & $\begin{array}{l}7,4 \% \text { (under } \\
35 \text { yy) }\end{array}$ & $\begin{array}{l}14,8 \% \text { (between } \\
36 \text { and 55yy) }\end{array}$ & $\begin{array}{l}18,5 \% \text { (between } \\
56 \text { and } 65 y y)\end{array}$ & $\begin{array}{c}33,3 \% \text { (between } 66 \text { and } \\
75 y y \text { ) }\end{array}$ & $\begin{array}{l}25,9 \% \\
\text { (over } \\
75 y y)\end{array}$ & \# & \# \\
\hline $\begin{array}{l}\text { Educational statement of patients re- } \\
\text { cruited expressed in percentage }(\%)\end{array}$ & $\begin{array}{l}37 \% \text { (primary } \\
\text { school) }\end{array}$ & $\begin{array}{l}33,3 \% \text { (junior } \\
\text { high school) }\end{array}$ & $\begin{array}{l}18,5 \% \text { (second- } \\
\text { ary school) }\end{array}$ & $11,1 \%$ (degree) & \# & $\#$ & $\#$ \\
\hline $\begin{array}{l}\text { Percentage (\%) distribution of patients } \\
\text { recruited in line with the the time- } \\
\text { course passed between brain damage } \\
\text { event and rehabilitative treatment }\end{array}$ & $11 \%$ (10 days) & $\begin{array}{l}30 \%(11-20 \\
\text { days })\end{array}$ & $\begin{array}{c}30 \%(21-30 \\
\text { days })\end{array}$ & $7 \%$ (31-40 days) & $\begin{array}{l}0 \%(41- \\
50 \text { days })\end{array}$ & $\begin{array}{c}7 \% \\
(51-60 \\
\text { days })\end{array}$ & $\begin{array}{l}15 \% \text { (more } \\
\text { than } 2 \\
\text { months) }\end{array}$ \\
\hline $\begin{array}{l}\text { Percentage (\%) distribution of patients } \\
\text { recruited in line with the brain damage } \\
\text { etiology }\end{array}$ & $8 \%$ (traumatic) & $30 \%$ (anoxic) & $\begin{array}{l}40 \% \text { (haemor- } \\
\text { ragic) }\end{array}$ & $22 \%$ (ischemic) & \# & \# & $\#$ \\
\hline $\begin{array}{l}\text { Limb distribution of spasticity ex- } \\
\text { pressed in percentage (\%) }\end{array}$ & $\begin{array}{l}15 \% \text { (nolimb- } \\
\text { spasticity) }\end{array}$ & $\begin{array}{l}41 \% \text { (regional- } \\
\text { limb spasticity) }\end{array}$ & $\begin{array}{l}44 \% \text { (general- } \\
\text { ized spasticity) }\end{array}$ & \# & \# & \# & \# \\
\hline $\begin{array}{l}\text { Spasticlimb pattern distribution ex- } \\
\text { pressed in percentage (\%) }\end{array}$ & $\begin{array}{l}22 \% \text { (unilateral } \\
\text { upper and low- } \\
\text { er limb spastic } \\
\text { flexed schema) }\end{array}$ & $\begin{array}{l}26 \% \text { (bilateral } \\
\text { upper and low- } \\
\text { er limb spastic } \\
\text { flexed schema) }\end{array}$ & $\begin{array}{l}26 \% \text { (bilateral } \\
\text { lower limb } \\
\text { spastic flexed } \\
\text { schema) }\end{array}$ & $\begin{array}{l}26 \% \text { (bilateral upperlimb } \\
\text { flexed schema and lower } \\
\text { limb spastic extension } \\
\text { schema) }\end{array}$ & $\#$ & \# & \# \\
\hline
\end{tabular}

\section{"Actual statement" impairment and clinical evaluation}

After the rehabilitative hospitalization, patients recruited were discharged with a "vegetative state" diagnosis and: - A LCF score $\leq 3$ (mean value $1,4 \pm 0.6$ at the first evaluation and 1,9 $\pm 0,6$ at the hospital discharge)

- A DRS score $\geq 22$ :

- A severe brain damage with a GCS score $\leq 8$ (mean value 5,7 $\pm 1,9)$.

$8 \%$ of all patients had a traumatic brain damage, $30 \%$ a post-anoxic damage, $40 \%$ ahaemorrhagic damage and $22 \%$ a post-stroke damage. We also analysedneuromotor impairment observing an $85 \%$ of patients affected by tetraplegia, $7.5 \%$ affected by hemiplegia and $7.5 \%$ by double hemiplegia. We didn't observe a variation of percentage of patients affected by tetraplegia while a decrease of percentage of patients affected by double hemiplegia was found when discharged from our hospital. 51\% showed a cardiovascular comorbidity, $4 \%$ infectious, metabolic and psychiatric diseases, $15 \%$ neurological comorbidities and $7 \%$ neoplastic disease.

\section{"Actual statement" spasticity evaluation}

$85 \%$ of patients recruited developed an upper and lower limb spasticity never demonstrated in other clinical evidences; $45 \%$ of them showed a moderate grade and $40 \%$ a severe grade of spasticity while $15 \%$ of patients never showed an upper and lower limb hypertonous. In a time-course evaluation of spasticity onset, we found that $48 \%$ of patients developed an early form of spasticity (present at the beginning of the hospitalization) and $37 \%$ a late form of muscle spasticity (developed during the recovery), while the remaining $15 \%$ never showed limb spasticity. We also analysed the limb distribution of spasticity. $41 \%$ of pa- tients developed a regional limb spasticity, 44\% a generalized form of spasticity and 15\% no limb spasticity. Different pathological spastic limb schema were observed during and at the end of the clinical and rehabilitative hospitalization. A flexed upper and lower limb spastic pattern was observed in $26 \%$ of patients recruited, 26\% developed a lower limb flexed schema only, $26 \%$ showed a bilateral flexed upper limb and a spastic extension lower limb pattern, while the remaining $22 \%$ of patients developed an upper and lower limb flexed schema only on a body side. Relationship between spasticity and brain damage etiology was analysed dividing patients into four observational groups: a) post-stroke group, post-haemorrhagic group, post-anoxic group, post-traumatic group. We observed that a) post-haemorrhagic group showed a higher incidence of limb spasticity, b) spasticity will develop earlier in post-stroke and traumatic patients c) spasticity severity is stronger in post-anoxic and traumatic brain injury. These parametric data were not statistically significant $(p>0.05)$. All patients underwent to drug and physiotherapist treatment and although $70 \%$ of them developed limb spasticity while $30 \%$ didn't show muscle tone changes. In line with the non- parametric statistical analysis, no correlation between the development of limb spasticity and nutritional and co-morbidity patients status was observed.

\section{Discussion}

The main findings of our investigational study are that (a) no clinical advanced evidence can be demonstrated about the pathophysiological mechanisms underlying the development of spasticity in patients affected by vegetative state secondary to severe acquired brain injury and (b) $85 \%$ of patients recruited in this study developed an upper and lower limb spasticity never 
demonstrated in other clinical evidences. We recruited in this study 27 patients, divided in 4 groups in line with the brain damage cause. In accordance with the results of the Italian GISCAR study ${ }^{[17,18]}$, we found that patients affected by severe brain damage secondary to vascular accidents represented the most numerous group of our investigational study (about 60\%). Discharged from our hospital with a "vegetative state" diagnosis, $40 \%$ of them had a haemorrhagic damage and $22 \%$ a post-stroke damage. The data was not in line with the literature, in which patients affected by a vegetative state secondary to an ischaemic brain damage were about 4\%only ${ }^{[18]}$. Although this, our result was not significant on an epidemiological point of view related with the smallness of the sample recruited.

$85 \%$ of our patients developed an upper and lower limb spasticity and underwent to a pharmacological and rehabilitative treatment. In an investigational point of view, this study suggested that a careful evaluation of patients affected SBI is needed in the pre-treatment phase to define their functional outcome is to optimize a task specific and integrated treatment approach of their limb spasticity development. Infact, we observed that post-haemorrhagic patients showed a higher incidence of limb spasticity and that spasticity severity is stronger in post-anoxic and traumatic brain injury. The best clinical strategy is to consider post-haemorrhagic and post-anoxic brain damage negative functional outcome parameters for patients affected by SBI and the matter to begin to treat immediately every kind of muscle overactivity in a strong and task specific way. No relationship between the development of limb spasticity and nutritional and co-morbidity patients' status was found in this study. It means that a good clinical governance and global nursing of needs of patients affected by SBI is often not enough to prevent the development of a dysfunctional muscle overactivity.

All patients recruited in this study underwent to a rehabilitative treatment. Although, we observed how critical review is needed to optimize or to individualize the treatment strategies in a cultural background in which only post-stroke rehabilitative course and practice are well known and usually applied in patients affected by SBI. In line with the conclusions of Malhotra, el al. ${ }^{[3]}$, we also think that the term spasticity is inconsistently defined and that the outcome measures considered in this study did not correspond the traditional academic definition of spasticity.

In conclusion, this observational and investigational study suggests that, to date, no clinical advanced evidence about the pathophysiological mechanisms underlying the development of spasticity in patients affected by vegetative state secondary to severe acquired brain injury can be demonstrated. Moreover, a comprehensive clinical and rehabilitative review is needed in order to optimize the complex management in patients affected by cognitive and motor disorders related to severe acquired brain injury.

\section{References}

1. Feldman, R.J., Young, R., Koella, W. Spasticity disordered motor control. (1980) Chicago: year book 185-204.

2. Lance, J., Feldman, R., Young, R., et al. Spasticity disordered motor control. (1980) Chicago: year book 51-55.

3. Malhotra, S., Pandyan, A.D., Day, C.R., et al. Spasticity, an impairment that is poorly defined and poorly measured. (2009) Clin Rehabil 23(7): 651-658.

4. Leong, B. The vegetative and minimally conscious states in children: spasticity, muscle contracture and issues for physiotherapy. (2002) Brain Inj 16(3): 217-230.

5. Sanger, T., Delgado, M., Gaebler-Spira, D., et al. Classification and definition of disorders causing hypertonia in childhood. (2003) Pediatrics 111(1): e89-e97.

6. Pandyan, A., Gregoric, M., Barnes, M., et al. Spasticity, clinical perceptions and neurological realities and meaningful measurement. (2005) Disabil Rehabil 27(1-2): 2-6.

7. Teasdale, G., Jennet, B. Assessment of coma and impaired consciousness. A practical scale. (1974) Lancet 2: 81-84.

8. Choi, S.C., Narayan, R.L., Anderson, R.L., et al. Enhanced specificity of prognosis in severe head injury. (1988) J Neurosurg 69(3): 381-385. 9. Starmark, J.E., Stalhammar, D., Holmgren, E., et al. A comparison of the Glasgow Coma Scale and the Reaction Level Scale. (1988) J Neurosurg 69(5): 699-706.

10. Jennett, B., Bond, M. Assessment of outcome after severe brain damage. (1975) Lancet 1: 480-484.

11. Gouvier, W.D., Blanton, P.D., LaPorte, K.K., et al. Reliability and Validity of the Disability Rating Scale and the Levels of Cognitive Functioning Scale in monitoring recovery from severe head injury. (1987) Arch Phys Med Rehabil 68(2): 94-97.

12. Hall, K., Cope, D.N., Rappaport, M. Glasgow Outcome Scale and Disability Rating Scale: comparative usefulness in following recovery from severe head injury. (1985) Arch Phys Med Rehabil 66(1): 35-37.

13. Bakheit, A.M., Maynard, V.A., Curnow, J., et al. The relation between Ashworth scale scores and the excitability of the alpha motor neurons in patients with post-stroke muscle spasticity. (2003) J NeurolNeurosurg Psychiatry 74(5): 646-648.

14. Pandayan, A.D., Price, C.I., Barnes, M.P., et al. Abiomechanical investigation into the validity of the modified Ashworth Scale as a measure of elbow spasticity. (2003) Clin Rehabil 17(3): 290-293.

15. Fleuren, J.F., Voerman, G.E., Erren-Wolters, C.V., et al. Stop using the Ashworth scale for the assessment of spasticity. (2009) J Neurol Neurosurg Psychiatry 81(1): 46-52.

16. Ghotbi, N., Ansari, N.N., Naghdi, S., et al. Inter-rater reliability of the Modified Ashworth scale in assessing lower limb muscle spasticity. (2009) Brain Inj 23(10): 815-819.

17. Klein, S., Kinney, J., Jeejeebhoy, K., et al. Nutrition support in clinical practice: review of published data and recommendations for future research directions. Summary of a conference sponsored by the National Institutes of Health, American Society for Parenteral and Enteral Nutrition, and American Society for Clinical Nutrition. (1997) Am J Clin Nutr 66(3): 683-706.

18. Zampolini, M., Zaccaria, B., Tooli, V., et al. Rehabilitation of traumatic brain injury in Italy: a multi-centred study. (2012) Brain Inj 26(1): 27-35.
Online ISSN: 2377-1348

Journal Title: International Journal Neurology and Brain Disorders Journal Short Name: Int J Neurol Brain Disord
Ommega Online Publishers

E-mail: neurology@ommegaonline.com

Website: www.ommegaonline.org 